\title{
A simple hands-off method for converting a biliary drain from mouth to nose
}

Endoscopic nasobiliary drainage (ENBD) is a widely used technique for bile duct decompression. It also provides access to the bile duct and allows cholangiography to be repeated. After placement, the drainage tube is left exiting from the mouth and re-routing via one of the nostrils is necessary. Conventionally, a nasopharyngeal tube, which is used as a carrier, is advanced into the patient's nose and into the posterior pharynx. The endoscopist then retrieves the tip of the tube in the pharynx with his fingers in the patient's mouth. This step causes discomfort to the patient and puts the endoscopist at risk of being bitten. A new method was reported recently using a pair of grasping forceps to capture the carrier through an end-viewing scope [1]. This method requires, of course, the use of an end-viewing endoscope. We describe here our novel method of extracting the carrier tube using a section of guide wire. A $3 \mathrm{~cm} \times 2 \mathrm{~cm}$ loop is made with a piece of guide wire (Jagwire; Boston Scientific Corporation, Miami, FL, USA) and inserted into the patient's oral cavity until it touches the posterior wall of the pharynx ( $\bullet$ Fig. 1). The endoscopist then loosens the wire loop, allowing it to occupy the width of the pharynx. A $16 \mathrm{~F}$ soft nasopharyngeal tube is advanced through the nose down to the pharynx. The tube can be easily trapped within the loop. When the wire is withdrawn, it pulls the carrier with it out of the mouth ( Fig. 2). A classical conversion is then performed to bring the drain out through the nose, using the carrier as the guide ( Video 1 ).

\section{Video 1}

A simple way to convert an orobiliary to a nasobiliary tube using a piece of guide wire.

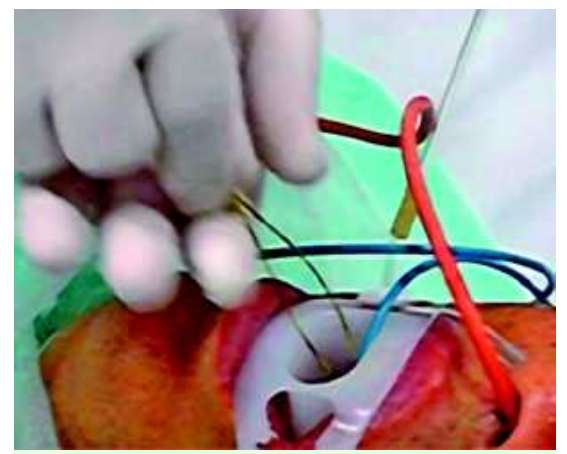

Fig. 1 A loop was made with a section of guide wire and inserted into patient's mouth.

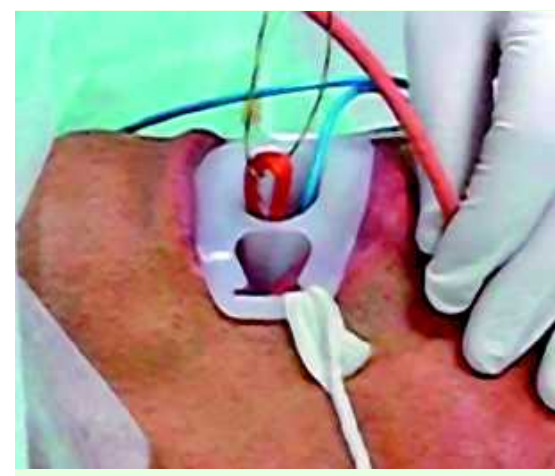

Fig. 2 The tip of the carrier was successfully extracted with the guide wire.

This method is quite simple and needs no additional accessories, as a used guide wire is readily available when ENBD is performed. The endoscopist does not require special training, and in our unit there is a more than $90 \%$ success rate at the first attempt. It takes only about 1 min to accomplish the conversion much shorter than the traditional way, which takes about 3-5 min. This method causes negligible discomfort to the patient and can be used when the patient is under conscious sedation even with the teeth-guard in situ. This novel technique is a simple, safe, convenient and timesaving method to convert an orobiliary to a nasobiliary tube.

\section{Endoscopy_UCTN_Code_TTT_1AR_2AK}

\section{R. Lu, S.-Z. Wang, B. Hu}

Endoscopy Center, Eastern Hepatobiliary Hospital, Second Military Medical

University, Shanghai, China

\section{Reference}

1 Shah SMA, Barkin JS. Conversion from an orobiliary to a nasobiliary tube: a hands-off method. Gastrointestinal Endoscopy 2007; 65: $355-356$

Bibliography

DOI 10.1055/s-2007-966864

Endoscopy 2009; 41: E11

(C) Georg Thieme Verlag KG Stuttgart · New York · ISSN 0013-726X

Corresponding author

\section{B. Hu, MD, PhD}

Endoscopy Center

Eastern Hepatobiliary Hospital

Second Military Medical University

225 Changhai Rd.

Shanghai 200438

China

Fax: + 8621-65562400

hubing@online.sh.cn 